\title{
Circulation et modélisation du discours testimonial dans le champ scolaire : l'exemple des manuels
}

The circulation and modelization of testimonial discourses: the example of textbooks

\section{Olivia Lewi}

\section{(2) OpenEdition Journals}

Édition électronique

URL : http://journals.openedition.org/aad/2438

DOI : 10.4000/aad. 2438

ISSN : 1565-8961

Éditeur

Université de Tel-Aviv

\section{Référence électronique}

Olivia Lewi, «Circulation et modélisation du discours testimonial dans le champ scolaire : l'exemple des manuels », Argumentation et Analyse du Discours [En ligne], 19 | 2017, mis en ligne le 15 octobre 2017, consulté le 23 septembre 2019. URL : http://journals.openedition.org/aad/2438 ; DOI : 10.4000/ aad. 2438

Ce document a été généré automatiquement le 23 septembre 2019

\section{cc)}

Argumentation \& analyse du discours est mis à disposition selon les termes de la licence Creative Commons Attribution - Pas d'Utilisation Commerciale - Pas de Modification 4.0 International. 


\title{
Circulation et modélisation du
} discours testimonial dans le champ scolaire : l'exemple des manuels

The circulation and modelization of testimonial discourses: the example of textbooks

\author{
Olivia Lewi
}

1 Travaillant sur le témoignage à travers le corpus particulier des récits de vie de survivants et de rescapés de la Shoah déposés au Centre de documentation juive contemporaine $^{1}$, j'ai pu constater que ces textes écrits par des scripteurs non professionnels, en marge des circuits éditoriaux, relèvent de scénographies singulières. $\mathrm{Si}$ « l'instauration de scénographies est indispensable quand il s'agit de capter un public, quand la prise de parole constitue, à des degrés divers, une prise de risque » (Maingueneau 2006), on comprend que l'acte de témoigner d'un événement exemplaire nécessite de légitimer son positionnement.

2 Pour cerner la scénographie ${ }^{2}$ propre aux textes de ce corpus, je m'intéresse à la «modélisation» et à la circulation discursive dont ils relèvent. En effet, ces témoignages sont «formatés " par d'autres textes, relevant de différentes scènes englobantes ${ }^{3}$ (littéraire, didactique), ce qui nous permet de faire apparaître des «modèles» de témoignage «qui exploitent de manière préférentielle certaines virtualités de cet hypergenre» (Maingueneau 2006). On peut définir l'hypergenre comme un « mode d'organisation textuelle élémentaire qu'on retrouve à des époques et dans des lieux les plus divers et à l'intérieur duquel peuvent se développer des mises en scène de la parole très variées » (Maingueneau $2006: 42$ ).

3 L'hypothèse selon laquelle l'école peut participer du processus de formatage du discours testimonial nous conduit à nous intéresser aux manuels scolaires susceptibles d'offrir un terrain favorable à l'observation de l'assimilation de schèmes narratifs. Si les manuels sont envisagés dans la première partie de l'analyse comme un moyen de formatage de l'écriture des scripteurs ordinaires, ils sont aussi, comme nous le verrons 
dans un second temps en nous appuyant sur les manuels d'histoire de Terminale ${ }^{4}$ générale récents, un des lieux privilégiés de l'expression de la doxa scolaire en matière de témoignage.

Pour mener cette réflexion, je mettrai en œuvre des outils théoriques de l'analyse du discours tels que les définit Dominique Maingueneau et j'emprunterai des notions à d'autres champs conceptuels, notamment la notion de circulation des discours qu'introduit Jeanneret (2008). Dans ce cadre, il s'agit «de repérer et d'analyser des facteurs de stabilisation et de légitimation pouvant conduire à la consécration testimoniale et ceux de mise à l'écart engendrant l'oubli » (Fleury et Walter 2014:8).

Pour constituer le corpus, j'ai retenu trois de ces textes, œuvres de témoins « ordinaires », non « consacrés » :

- Anonyme (Musia). 1980. Sans titre (désormais texte 1)

- Elias Quddus Marguerite. 1998. Cachée (désormais texte 2)

- Anonyme. 1998. Le témoin sursitaire. Récit autobiographique (désormais texte 3).

Les éléments proprement biographiques concernant ces auteurs sont très lacunaires, notamment en ce qui concerne les textes 1 et 3. L'auteure du texte 2 est issue d'une famille juive parisienne d'origine russe et a été cachée pendant la guerre en Isère. Elle précise en avant-propos de son récit : «Mon récit commence en 1941, suivi d'un court retour en arrière sur l'exode de Paris. Je reconstruis l'image historique du 99 rue de Charonne. Je revis cette époque, recréant l'ambiance de mon enfance, du bonheur, à l'absence de celui-ci, mes joies, mes peines, mes peurs, mes révoltes face à l'injustice et l'incompréhension, les adaptations forcées successives, puis l'acceptation inévitable de la tutelle de remplacement, avec le sens de culpabilité qui en a résulté.»

7 Je me demanderai par quelles opérations normatives, liées à la scène englobante didactique, l'école construit la figure d'un "lecteur modèle » - selon l'expression d'Umberto Eco (1979) - du discours testimonial, et dans quelle mesure elle participe en cela du processus de patrimonialisation de ce type de discours. J'analyserai tout d'abord ce phénomène de modélisation observable dans certains récits de rescapés à partir du cadre normatif des rédactions scolaires proposées dans les manuels de «cours de langue française » des années 30 .

\section{La captation de la matrice rédactionnelle des manuels de français}

8 Parmi les tapuscrits déposés au Mémorial de la Shoah à Paris, les plus récents (datant des années 1980 à 2000) sont constitués de récits de rescapés et majoritairement de récits d'enfants cachés ${ }^{5}$. Au moment de la guerre, ces " écrivants» (selon l'expression de Roland Barthes) - car c'est très souvent le statut qu'ils revendiquent - avaient entre 5 et 14 ans et, pour ce qui concerne ce corpus, ils ont été scolarisés pour la plupart en France.

9 Le motif narratif des scènes d'école qui s'y trouve de manière récurrente renvoie à la découverte de l'antisémitisme et bien souvent à celle de la rupture avec l'institution, étant donné l'impossibilité de poursuivre sa scolarité. Les traces du modèle scolaire de rédaction tel qu'il était enseigné à l'école primaire dans les années 30-40 peuvent être interprétées comme le signe d'une volonté de «réinscription" sociale à travers l'institution. En effet, « ce modèle ne représente pas [...] la simple trace stylistique d'un 
apprentissage spécifique de l'écriture; il gouverne, semble-t-il, le récit de vie à un niveau plus profond " (Chanfrault-Duchet 1985: 83). Cette réinscription sociale s'effectue, comme je me propose de le montrer, par le biais d'une « captation », c'est-àdire par le choix d'une stratégie de "réinvestissement d'un texte ou d'un genre de discours dans d'autres » (Maingueneau 2002 : 93).

\subsection{Les exercices d'écriture : un " préconstruit ${ }^{6}$ dans les récits de vie des rescapés}

10 Dans la mesure où les auteurs ne cherchent pas à faire œuvre littéraire, l'écriture privée ne dissimule pas la tendance à reproduire celle qui s'enseigne à l'école. Le geste autobiographique vient ainsi coïncider avec le rituel de la rédaction scolaire, exercice qui sollicite constamment le vécu de l'élève puisqu'il consiste majoritairement à prescrire la mise en récit des anecdotes personnelles.

11 Ainsi, on retrouve dans les textes du corpus un certain nombre de patrons narratifs proposés aux élèves dans les exercices de « composition » : description de lieux (décors familiers ou trajets), jeux d'enfants, scènes d'école, scènes de lecture, portrait physique de personnages, expression des affects comme la peur, sensations liées à la nature, au réveil ou au coucher de l'enfant.

Le modèle scolaire m'a semblé d'autant plus évident que trois des manuels consultés proposent des consignes de rédaction auxquelles semblent répondre les textes analysés :

Vous êtes dans votre lit le matin. Vous écoutez les bruits qui vous parviennent, bruits de la maison qui se réveille, bruits de la vie qui reprend. Notez ces bruits depuis votre réveil jusqu'à votre lever (Delagrave : 1935).

Composition française : Le jour naît. Jour de travail : le devoir à accomplir, pensées, résolutions (Belin : 1940).

Traitez le sujet contraire : « La maison s'éveille ». Des bruits rompent le silence : par exemple des pas qui s'éloignent sur la route...L'aube dissipe la nuit. Des chants d'oiseaux saluent les premières lueurs du jour. Soudain, des bruits venus de la maison...Lesquels? Et voici la lumière...et le jour triomphant (Charles-Lavauzelle : 1947).

13 Ces exercices suivent un même extrait soumis à la lecture des élèves et destiné à être imité. Il s'agit d'un ouvrage de G. Duhamel, Les plaisirs et les jeux (Delagrave 1935 : 32) :

Une mouche, encore somnolente, traverse la chambre à l'aveuglette, se heurte au mur, bourdonne avec rage et se rendort.

Du fond de l'infini, un bruit régulier comme celui d'une horloge, plus marqué de seconde en seconde : un pas sur la route, le pas de l'ouvrier matinal; des coups sourds, pesants et, par-dessous, le crépitement du gravier meurtri. Le pas approche; dans un coin de la chambre, un objet attentif vibre délicatement, au rythme du marcheur. Puis le pas s'évanouit, comme s'il avait tourné de l'autre côté du monde.

Qu'est-ce donc? La nuit à son tour semble fissurée, blessée. Trois images bleues émergent des ténèbres. Les fenêtres! L'aube, l'aube! Si pâle qu'elle ne pourra jamais venir à bout de tout ce noir...

Un petit oiseau se met à chanter, tout seul, dans le marronnier. Il est au sommet des ramures. Sa chanson, toute ébouriffée, tombe en étendant les ailes.

L'homme écoute, écoute. Son corps se rassemble autour de lui, comme l'équipe des tâcherons à l'appel du métayer. Présent ! Présent !

Et, tout à coup, venue des entrailles de la maison, une petite voix humaine, nette, mélodieuse, dansante, prononce des mots que l'on ne comprend pas. Une autre voix 
lui répond, aussi faible, aussi pure, aussi joyeuse. Les deux voix s'emmêlent, s'enrouent, s'enlacent, s'élancent. Cris, rires, chants !

Toute la maison s'étire, gronde et fait le gros dos. Les enfants sont réveillés. Les enfants! Les enfants!

Victorieuse, la lumière se déverse dans l'âme, la lumière semblable aux eaux d'une cataracte.

Un jour à vivre !

Les similitudes constitutives d'une telle « matrice discursive » " peuvent être de l'ordre de la représentation [...]. Elles peuvent aussi être de nature linguistique : il s'agit alors, par exemple, de repérer les diverses formes d'actualisation des opérations énonciatives » (Beacco, Charaudeau et Maingueneau 2002 : 367).

Ainsi, la comparaison de l'incipit du texte 1 de notre corpus avec le texte de Duhamel permet de mettre en évidence des choix énonciatifs similaires :

«Wojna! Wojna! La guerre! La guerre !...»

La voix du speaker parvenait de loin aux sens encore endormis de Musia. Il faisait bon dans le vaste lit tiède et douillet de ses parents et c'est à contrecœur qu'elle laissait ces mots étranges l'envahir, excitant sa curiosité. Qu'est-ce que cela voulait bien dire ? Elle entrouvrit un œil et se réveilla tout à fait. Décidément, il se passait un événement inhabituel. Une atmosphère d'appréhension planait dans la chambre, d'ordinaire si paisible. Les ombres dansaient sur les murs, la lampe de chevet clignotait et la radio déversait un tas de mots dont le sens échappait complètement à Musia, tandis que son père, penché sur l'appareil, écoutait attentivement, inquiet, la main sur le menton.

16 Bien évidemment, la scène d'énonciation est très différente de celle du texte-source, mais le positionnement énonciatif imposé au scripteur non expert auquel on demande de s'inspirer des modèles littéraires rejoint celui de Duhamel : on note une hésitation énonciative qui se traduit à la fois par le choix d'une mise à distance du «je » autobiographique, l'utilisation de la troisième personne, point focal central des sensations, et par le recours au discours indirect libre. Cette modalité énonciative se manifeste dès l'avant-propos :

Voici un récit sans prétention littéraire.

C'est l'histoire authentique et intégrale d'une enfant dans cet enfer humain qu'est le racisme. Elle aurait pu aussi bien être l'histoire d'un million d'autres gosses. La seule différence entre eux et Musia, c'est qu'elle survécut.

On constate ici, comme le souligne Bishop (2010: 52) à propos des positions énonciatives attendues des élèves face aux sujets de rédaction qui leur sont soumis, que

[t]rois positions énonciatives découlent de cette demande d'écriture. Celle du personnage qui, en se racontant, répond au contrat autobiographique. Celle de l'observateur, centre de focalisation, et celle de l'évaluateur qui nécessite une mise à distance et une évaluation de la situation. [...] La flexibilité dans l'emploi des modes de présentation et des pronoms semble faire partie des critères de réussite.

18 La prégnance des modèles littéraires présentés dans les manuels se manifeste également dans l'incipit du texte 2 de notre corpus :

Douillettement blottie, au creux de ma forteresse de coussins en plumes, je me repose, pour soigner mon rhume.

Une chaude caresse me chatouille le bout du nez, et des picotements multicolores traversent mes paupières closes.

Je dors, je dors, je dors, je dors... Car si je veux sortir dehors, je dois obéir d'abord.

La T.S.F. fredonne : « La rue de nos amours... On y voit rôder le soir, des amoureux dans les coins noirs... » Chanson que mes parents adorent, valsant dans mon décor ! Les ronflements de la machine à coudre de maman ont repris, plus forts qu'avant, 
Rrr...Rrr...Rrr...L'un derrière l'autre, en queue-leu-leu. Ils font vibrer les pieds des fauteuils, au centre desquels on m'a installée, en face de la fenêtre ouverte. Et comme les feuilles des arbres dans le vent, mes draps frissonnent mollement, à chacun d'eux.

Je me laisse bercer dans le train imaginaire...Sur la route familière, du conducteur effréné. Les wagons n'en finissent pas de passer.

Au rez-de-chaussée, la locomotive gronde, et, dans sa lancée, je vagabonde, aux pays des songes. Mes histoires s'allongent, m'empêchant d'oublier que l'on travaille à l'atelier.

STOP ! Tout le monde descend ! Les tremblements ont cessé, le bruit s'est arrêté, lui aussi. Seule la musique continue son chemin mélodieux. Je ne veux plus fermer les yeux, j'appelle « Maman! Maman!». Personne ne m'entend.

Le rapprochement avec l'extrait du Voyage égoïste de Colette montre une proximité patente :

Malade. Oh! Oui, enfin malade. Un peu de grippe, sans doute ? Je referme les yeux et j'attends le commencement de cette journée de faiblesse, de demi-sommeil, de caprices respectés, de diètes gourmande...

Faut-il attendre que la maison s'éveille? Ou bien sonnerai-je pour qu'on se hâte et qu'on s'effare, avec des bruits de mules claquantes dans l'escalier, des «Mon Dieu!» et des «Cela devait arriver, la grippe court... ». Mieux vaut attendre, en guettant le jour qui grandit, le tapis s'éclaire et pâlit comme une mare... J'entends, mais vaguement, le roulement des voitures et les sonnailles des bouteilles pendues aux doigts du laitier... Le son profond d'une timbale grave, battue légèrement et régulièrement, assourdit mes oreilles et me sépare des bruits de la rue; c'est la monotone, l'agréable pédale de ma fièvre....

Quoi ? Que voulez-vous ? Je dormais...oui, vous voyez, je suis malade...Si, si vraiment malade !... (Delagrave $1935: 56$ )

Le choix énonciatif de la narratrice du texte 2 rejoint celui de Colette dont elle semble adopter l'énonciation enfantine en focalisation interne. Il s'agit de répondre à l'injonction d'authenticité, de "faire vrai», comme le recommande par exemple le manuel aux éditions Charles-Lavauzelle dans les termes suivants : « Il faut [...] que, dans vos narrations, les actions et les sentiments des personnages soient naturels ».

21 L'incipit du texte 3 met lui aussi en avant une structure similaire à celle du texte de Colette ainsi que la mise en scène d'un « je », point focal des sensations :

Je suis fiévreux, front brûlant, les oreilles bourdonnantes et je flotte, dans une somnolence ouatée, oscillant entre l'éveil et l'inconscience. Je balaie du regard le plafond et les murs de l'unique grande pièce qui nous sert, tout à la fois, de salle à manger et de chambre avec un grand lit pour mes parents, séparé du mien par un paravent. Par sa porte ouverte, j'aperçois la petite cuisine, éclairée par un vasistas percé dans le toit incliné, dont l'évier nous permet aussi la toilette.

Mes yeux se ferment, tellement mes paupières sont lourdes, et j'en viens à rêver à je ne sais plus qui ou quoi aujourd'hui avec, en fond, un ronronnement apaisant. Une anesthésie progressive, en quelque sorte. Le ronronnement s'amplifie démesurément, à tel point qu'il devient le rêve, balayant tout le reste. Brusquement des coups sourds l'accompagnent pour le rythmer, et ce martèlement agresse mes tympans. Je me réveille en sursaut... et je prends conscience que le ronronnement et les coups de mon rêve sont bien réels car il s'agit de la sonnette de la porte palière et de poings fermés qui la martèlent. D'une voix éraillée, que j'essaie tant bien que mal d'assurer pour faire cesser ce vacarme, je crie «Voilà, j'arrive! ». Je me lève, titubant quelque peu, me dirige en pyjama vers la porte et demande « Qui est là?».

Dans les trois textes, la doxa scolaire se manifeste également dans la présence, plus ou moins insistante selon les récits, d'expressions stéréotypées comme ces syntagmes 
axiologiquement marqués : "lit tiède et douillet ", " une chaude caresse ", "sur la route familière ", « son chemin mélodieux » (texte 2) ; « une somnolence ouatée », « un ronronnement apaisant ", "une voix agréable et douce " (texte 3). Ces expressions trahissent une référence commune à un discours dont les codes sont connus du lecteur qui les a, comme le scripteur, assimilés au cours de sa scolarité.

De même que le respect de la doxa scolaire et des normes de l'institution permet à des auteurs non professionnels visant la reconnaissance et la consécration sociale d'autoriser leur parole et de légitimer leur récit (Chanfrault-Duchet 1985), l'authenticité des paroles de celui qui fait l'expérience de la mise au ban l'impose dans l'espace social comme un témoin légitime.

\subsection{Doxa scolaire et dimension sociale} concept de "témoin modèle » à celui d'un « lecteur modèle ». La notion de modèle s'avère en effet centrale pour comprendre le fonctionnement de l'hypergenre du témoignage et en particulier la scénographie des récits du corpus. Envisager le témoignage comme un hypergenre permet d'inscrire les productions singulières dans un ensemble plus vaste, en fonction de l'événement qui les cadre et les autorise, car les modes de fabrication et de façonnage du discours testimonial sont le fruit d'interrelations constantes entre les composantes collectives et individuelles. Ainsi, la posture de «témoin modèle » en attente d'un co-énonciateur variant en fonction de la façon dont il s'inscrit dans la problématique mémorielle du moment de l'énonciation, le concept de « lecteur modèle » est-il « construit sur la base d'indices variés, mais il n'est pas nécessaire qu'il corresponde à une représentation consciente chez le producteur : il 
fait partie intégrante de la définition d'un genre de discours ou d'un positionnement " (Maingueneau $2002: 338$ ).

Et, comme je l'ai développé ailleurs (Lewi 2017),

[si] l'énonciation du témoin-survivant nécessite d'être ancrée dans l'authenticité fabriquée des paroles de ceux qui ont vécue l'événement traumatique, ce que met en avant l'instabilité de la posture énonciative du scripteur «non professionnel» [...], c'est le fait que la réalité évoquée, bien que temporellement proche reste difficilement énonçable et donc représentable. La question n'est pas seulement celle de l'indicible mais plutôt celle d'un positionnement encore problématique de l'énonciateur en attente d'un co-énonciateur dont la compétence reste à définir.

On voit ainsi se dessiner une boucle réflexive ${ }^{9}$ : le discours testimonial des enfants cachés s'inscrit en partie dans un canon scolaire qui leur permet d'acquérir une certaine légitimité. En intégrant ce canon ils participent en retour à la normalisation de ce discours, à sa modélisation et à sa circulation.

\section{L'intégration des témoignages dans les manuels d'histoire}

\subsection{Témoins « ordinaires », témoins consacrés}

L'observation des textes de témoignage dans les manuels d'histoire de Terminales générales, amène à s'interroger sur la manière dont l'institution scolaire « consacre » véritablement la parole de certains témoins.

31 Seul le manuel Hatier (lequel offre le plus grand nombre de témoignages comme documents) donne à lire le récit de trois témoins «ordinaires». Dans les autres manuels, la grille de lecture proposée aux élèves souligne la légitimité à la fois sociale et intellectuelle des témoins choisis :

Simone Veil, rescapée d'Auschwitz, présidente d'honneur de la Fondation pour la mémoire de la Shoah. (Manuel Belin $2012: 87$ )

Ancienne ministre et survivante d'Auschwitz, Simone Veil est présidente d'honneur de la fondation pour la mémoire de la Shoah. (Manuel Nathan 2012 : 70)

L'ethnologue Germaine Tillion fut déportée à Ravensbrück en tant que résistante. Pendant son internement puis lors de la libération du camp, elle eut recours à des méthodes scientifiques pour appréhender l'univers concentrationnaire. (Manuel Hatier)

Déposition de Marie-Claude Vaillant-Couturier devant le tribunal de Nüremberg, 28 janvier 1946. «Une résistante, arrêtée en 1942 par la police française et internée à Auschwitz, témoigne ». (Manuel Hachette $2012: 62$ )

Grande figure féminine de la Résistance, Lucie Aubrac a combattu contre les guerres coloniales et en faveur de la cause des femmes. Témoin lors du procès de Klaus Barbie, elle multiplie les interventions dans les établissements scolaires, dont plusieurs portent aujourd'hui son nom. (Manuel Nathan $2012: 69$ )

Les facteurs contribuant à l'accès au statut de témoin «nécessaire » ou « incontournable » peuvent être caractérisés en lien avec le statut social, le capital culturel, l'exemplarité ou la singularité de l'expérience: Auschwitz, Ravensbrück, l'engagement dans la résistance, la participation à des institutions reconnues comme la Fondation pour la mémoire de la Shoah. 


\subsection{Fonctionnement de la fabrique du « témoin modèle »}

un point de vue quantitatif, les documents désignés comme "témoignages » dans les chapitres consacrés à la lecture historique des mémoires ${ }^{10}$ et en particulier dans ceux qui sont consacrés au rapport entre l'historien et les mémoires de la Seconde mondiale en France ne constituent qu'un dixième des documents proposés aux élèves et ils font l'objet à chaque fois qu'ils apparaissent d'une décontextualisation du fait du paratexte et de l'appareillage didactique. Ces textes sont en effet détachés du contexte d'écriture auquel ils se rapportent et la fonction qu'ils jouent dans la vie du scripteur n'est plus la même. Comme le souligne Delormas (2017) à propos des anthologies littéraires,

[1]a vocation première des textes dans lesquels les extraits ont été prélevés n'apparaît plus, formatés pour les besoins d'une cause ; transformés par la découpe opérée dans le texte source, les extraits dans l'anthologie obéissent à des codes et à des normes nouvelles qui sont celle d'un manuel.

Lyon-Caen et Ribard (2010: 51) dénoncent ce problème dans la communauté des historiens lorsqu'elles écrivent que

[1] historiens n'héritent pas seulement de témoignages, tenus ou non comme littéraires à évaluer : il arrive qu'ils les constituent en désignant certains écrits comme des témoignages : des écrits de toutes sortes, des plus personnels (journaux intimes non publiés) aux plus littéraires (romans), sont dans ce cas mobilisés, souvent découpés et contextualisés de manière à les faire témoigner.

Ainsi pour mettre en avant sa valeur de document historique, le témoignage de Laurent Lazare Golberg ${ }^{11}$, dans le manuel Hatier (2012 : 18) destiné aux classes de Terminales L/ ES, intègre-t-il l'article du Journal officiel mentionnant la déchéance de nationalité sous Vichy :

Mes parents, originaires de Pologne, avaient demandé la nationalité française et l'avaient obtenue au terme de longues démarches, en 1938. Nous étions officiellement citoyens français, fiers d'appartenir pleinement à notre nouvelle patrie. L'adage «Heureux comme Dieu en France », cher au cœur des immigrés venus d'Europe de l'Est, prenait tout son sens [...]. Mais la guerre a tout bouleversé. Mon frère a été appelé sous les drapeaux et a combattu pour la France. Et puis est venu le temps de la défaite, l'instauration d'un nouveau gouvernement et l'adoption de lois antijuives. Le nouveau régime n'a pas hésité à revenir sur les décisions adoptées avant-guerre. C'est ainsi que nous avons pu lire, dans le Journal officiel : « Ministère de la Justice, décret $\mathrm{n}^{\circ} 3271$ du 4 novembre 1942 , portant retrait de la nationalité française.

Nous, Maréchal de France, chef de l'Etat français, [...] décrétons : Art $1^{\mathrm{er}}-$ La qualité de français est retirée à Golberg (Lejb) [...] naturalisé français par décret du 3 mars $1938,[\ldots] »$.

Les énoncés "greffés » selon l'expression de Bordron (2014:53) participent ainsi à cette « normalisation » du discours testimonial, ils ont une fonction « modélisante ». En effet, la présence importante dans le cotexte immédiat ou plus large des témoignages d'énoncés qui relèvent du champ juridique tend à souligner la fonction d'attestation du témoignage ${ }^{12}$. On peut le constater également dans les frises chronologiques mettant en avant les dates des différents procès (Papon, Barbie), les documents iconographiques représentant ces procès, la présence d'une « déposition » au procès de Nuremberg et le lien biographique fort de Simone Veil avec l'univers juridique dans la représentation collective. Cela est évidemment à rapprocher de la place des procès dans la construction des mémoires de la Seconde guerre mondiale et en particulier du procès Eichmann, événement historique décisif des années 1960, qui a initié le récit collectif et 
médiatisé de la Shoah et l'avènement du témoin dans l'espace public (Wievorka 1998 : 81). ci dans l'espace social se traduit par une instabilité énonciative du témoignage qui a partie liée avec sa malléabilité, sa disponibilité discursive. Cela contribue à renforcer l'hypothèse selon laquelle la capacité du discours testimonial à se fondre dans d'autres genres de discours ou à être formaté par eux est bien le signe de son appartenance à un hypergenre.

Le rapprochement des trois textes analysés souligne la fonction de légitimation qu'exerce la doxa scolaire sur le discours testimonial par le biais des manuels. Les scripteurs-témoins « ordinaires » comme les concepteurs des manuels obéissent à une représentation sociale bien établie de ce qu'ils pensent être un témoignage lorsqu'ils mettent en avant son authenticité et sa visée explicative. Ce "lissage " modélisant s'effectue soit sur le mode de la captation d'un genre de discours préconisée dans les manuels de littérature, soit sur celui de l'intégration au sein du discours didactique des manuels d'histoire. La légitimité ainsi revendiquée ou offerte au discours testimonial se négocie au prix d'une évidente uniformisation.

Argumentation et Analyse du Discours, 19 | 2017 


\section{BIBLIOGRAPHIE}

\section{Manuels}

Cours de langue française. Vocabulaire-Grammaire-Composition française, à l'usage des cours complémentaires des écoles primaires supérieures, des écoles pratiques de commerce et d'industrie, 1935

(Paris : Delagrave)

Leçons de français, Vocabulaire, Grammaire et composition française par l'étude des textes, Cours supérieur 2ème année, cours préparatoire des E.P.S. et cours complémentaires, 1940 (Paris : Belin)

Les textes littéraires, Lecture et récitation, exercices de rédaction, Cours de fin d'études primaires, 1947

(Paris : Charles-Lavauzelle)

Billard, Hugo (éd.). 2012. Histoire Terminales ES, L. Regards historiques sur le monde actuel (Paris : Magnard)

Bourel, Guillaume \& Marielle Chevallier. 2012. Histoire Terminales L-ES, Regards historiques sur le monde actuel, Manuel de l'élève (Paris : Hatier)

Colon, David (éd.). 2012. Histoire Terminales L, ES, Manuel de l'élève (Paris : Belin)

Cote, Sébastien (éd.). 2012. Histoire Terminales L-ES, Manuel de l'élève (Paris : Nathan)

Zachary, Pascal \& Vincent Adoumié (éds.). 2012. Histoire Terminales ES/L. Regards historiques sur le monde actuel, Manuel de l'élève (Paris : Hachette)

\section{Bibliographie générale}

Amossy Ruth. 1999. Images de soi dans le discours. La construction de l'ethos (Paris : Delachaux \& Niestlé) Amossy Ruth. 1999. Images de soi dans le discours. La construction de l'ethos (Paris :

Delachaux \& Niestlé)

Amossy Ruth. 1999. Images de soi dans le discours. La construction de l'ethos (Paris : Delachaux \& Niestlé)

Amossy Ruth. 2004. «L'espèce humaine de Robert Antelme ou les modalités argumentatives du discours testimonial », Semen 17

Amossy Ruth. 2010. La présentation de soi. Ethos et identité verbale (Paris : PUF)

Bishop, Marie-France. 2010. « Racontez vos vacances... ». Histoire des écritures de soi à l'école primaire (1882-2002) (Grenoble : Presses universitaire de Grenoble)

Bordron, Jean-François. 2014. : « Figures du détachement », Cossutta Frédéric \& Cicurel Francine (éds). Les formules philosophiques (Limoges : Lambert-Lucas), 53-69

Charaudeau, Patrick \& Dominique Maingueneau. 2002. Dictionnaire d'analyse du discours (Paris : Seuil)

Chaufrault-Duchet, Marie-Françoise. 1985. « La doxa scolaire dans les récits de vie » Cahiers de sémiotique textuelle 4, «Récits de modèles et écarts »,79-94

Coquio, Catherine. 2003 "L'émergence d'une "littérature" de non-écrivains : les témoignages de catastrophes historiques ", Revue d'histoire littéraire de la France 103, 343-363

Delormas, Pascale. 2014 « Espace d'étayage : la scène et la coulisse », in Sergier M., Vandevoorde H. \& van Zogge M. (éds), Over de auteur / À propos de l'auteur, CLW 6 (Gand : Academia Press) 
Delormas, Pascale. 2017 (à paraître). « L'anthologie littéraire : un genre didactique citationnel ». Le discours rapporté. Temporalité, histoire, mémoire et patrimoine discursif (Paris : Classiques Garnier)

Dulong, Renaud. 1998. Le témoin oculaire. Les conditions sociales de l'attestation personnelle (Paris : Ed. de l'EHESS)

Eco, Umberto. 1979. Lector in fabula. Le rôle du lecteur ou la coopération interprétative dans les textes narratifs (Paris : Grasset)

Kuentz, Paul. 1972. L'envers du texte, Littérature 7, 3-26

Jeanneret, Yves. 2008. Penser la trivialité, La vie triviale des êtres culturels (Paris : Hermès-Lavoisier)

Lacoste, Charlotte. 2011. Le témoignage comme genre littéraire en France de 1914 à nos jours, Thèse en sciences du langage et Littérature comparée

Lewi, Olivia. 2017 (à paraître). « Rôle et usage du discours rapporté dans la fabrique de l'éthos du témoin-survivant : l'exemple des récits de vie déposés au Mémorial de la Shoah (de 1945 à nos jours) ». Le discours rapporté. Temporalité, histoire, mémoire et patrimoine discursif (Paris : Classiques Garnier)

Lyon-Caen, Judith \& Dinah Ribard. 2010. L'historien et la littérature (Paris : La Découverte)

Maingueneau, Dominique. 1979. Les livres d'école de la République, 1870-1914 : discours et idéologie (Paris : Le Sycomore)

Maingueneau Dominique. 1999. «Ethos, scénographie, incorporation », R. Amossy (éd.) Images de soi dans le discours. La construction de l'ethos (Paris : Delachaux \& Niestlé), 75-100

Maingueneau, Dominique. 2004. Le Discours littéraire. Paratopie et scène d'énonciation (Paris : Colin)

Maingueneau, Dominique. 2006. «Le dialogue comme hypergenre », Ph. Guérin (éd.). Le dialogue ou les enjeux d'un choix d'écriture (Rennes : Presses Universitaires de Rennes), 35-49

Maingueneau, Dominique. 2013. «L'èthos : un articulateur », COnTEXTES 13, en ligne : https:// contextes.revues.org/5772

Waintrater, Régine. 2003. Sortir du génocide (Paris : Payot)

Wieviorka, Annette. 1998. L’Ère du témoin (Paris : Plon)

\section{NOTES}

1. Il existe, au Centre de documentation juive contemporaine, un fond de tapuscrits dont la collecte a commencé dans les années 1990 et continue aujourd'hui, constitué d'une trentaine de boîtes contenant des ouvrages variés dans différentes langues provenant essentiellement d'archives familiales données au Centre. Quelques-uns de ces tapuscrits ont été ensuite édités. Nous avons choisi de ne sélectionner que les récits pour l'essentiel autobiographiques écrits en français non publiés à ce jour, ce qui constitue une vingtaine de tapuscrits.

2. "Scène de parole que le discours présuppose pour pouvoir être énoncé et qu'en retour il doit valider à travers son énonciation » (Maingueneau $2004: 192)$.

3. La "scène englobante" est le cadre socio-discursif dont relève un genre de discours (Maingueneau $2004:$ 191).

4. Troisième et dernière année de lycée.

5. Ce corpus représente environ un tiers du corpus total des tapuscrits retenus pour ma recherche. 
6. «Le préconstruit peut être approché comme la trace, dans l'énoncé, d'un discours antérieur ; il s'oppose donc à ce qui est construit au moment de l'énonciation. Un sentiment d'évidence s'attache au préconstruit parce qu'il a été "déjà dit" et qu'on a oublié qui en était l'énonciateur. [...] La notion de préconstruit est étroitement liée à celle d'interdiscours » (Branca-Rosoff, Charaudeau et Maingueneau, $2002: 464$ ).

7. Cours de langue française 1935, Leçons de français 1940, Les textes littéraires 1947. Cf. bibliographie.

8. «L'ethos collectif correspond à une vision globale, mais à la différence de l'ethos singulier, il n'est construit que par attribution apriorique, attribution d'une identité émanant d'une opinion collective vis-à-vis d'un groupe autre » Charaudeau (2005: 90).

9. «Un discours impose sa scénographie d'entrée de jeu ; mais d'un autre côté l'énonciation, en se développant, s'efforce de justifier son propre dispositif de parole. On a donc affaire à un processus en boucle » (Maingueneau $2002: 516$ ).

10. http://cache.media.eduscol.education.fr/file/H-G_2015/96/4/Ress_Hist_TermESL_Theme1_memoires_503964.pdf

11. Témoignage présenté dans Mémoires de la Shoah 1933-1946 photographies et témoignages, Editions du Chêne Livre, 2005.

12. "Le témoignage ne serait pas seulement la description d'un état du monde, mais un acte de langage par lequel le locuteur s'engage personnellement, en premier lieu sur sa légitimité de témoin - “j'y étais" -, en second lieu sur la véracité de ses propos - "cela s'est effectivement passé" - (engagement qui, au tribunal, est pris sous serment et peut être légalement sanctionné) » (Amossy 2004).

13. J'adopte ici une conception élargie de la notion d'espace d'étayage qui, tel que défini par Delormas (2014) «permet de donner une certaine visibilité à la fabrique de l'image auctoriale, la figure d'auteur, indissociable de l'œuvre, n'émergeant pas de la seule œuvre mais de tout l'interdiscours, c'est-à-dire, par exemple, des commentaires critiques qui la promeuvent ou la discréditent et qui donnent lieu à la reconnaissance collective dont l'œuvre a besoin pour exister et circuler».

14. "Se déclarer témoin d'un fait, même dans un contexte informel, fait signe vers l'espace juridique et ses formules rituelles », rappelle Renaud Dulong (1998 : 57).

15. «Pour mener à bien cette mission d'éducation qui justifie leur survie, les témoins ne s'en tiennent donc pas à l'admonestation, au " plus jamais ça ! ... ». Assumant une fonction de guides, ils entendent contribuer à prévenir le retour du même en instruisant le lectorat. [...] Les textes testimoniaux se sous-tendent donc d'un vrai souci didactique » (Lacoste 2011 : 767).

\section{RÉSUMÉS}

Cet article se propose d'aborder la question du témoignage sous l'angle de l'analyse du discours en s'intéressant plus particulièrement au processus de "modélisation » du discours du témoin dans le champ scolaire. On assiste en effet à un «conditionnement du lisible» (Kuentz 1972), visant à faire de ce discours, a priori dénué de toute intention argumentative, un discours modélisé et qui fournit à son tour un certain modèle discursif. Nous développerons deux exemples d'opérations normatives en envisageant tout d'abord cette «modélisation » sous l'angle du lissage discursif à travers la reprise de modèles scolaires de rédaction dans un corpus constitué de récits de vie déposés au Mémorial de la Shoah. Puis nous nous pencherons sur la 
place faite aux témoignages et sur la fabrique du "témoignaire " (Waintrater 2003) dans les manuels scolaires d'histoire et nous développerons le concept de « témoin modèle ».

This paper intends to tackle the question of testimony in terms of discourse analysis with a focus on the "modeling" process of witness discourse in the school field. We observe a "conditioning of readable" (Kuentz 1972) that changes witnesses' speeches, without argumentative intention, into a modelled discourse and which works in its turn a certain discursive model. We will develop two examples of normative operations by first of all considering this "modeling" under the angle of discursive smoothing, through the rework of school patterns in in the life narratives left in Shoah Memorial in Paris. Then we will study the place let to testimonies and the factory of the "témoignaire" (Waintrater 2003) in history's school handbooks of Final and we will develop the concept of "model witness"

INDEX

Mots-clés : matrice discursive, modèles scolaires, modélisation, témoignage

Keywords : discursive matrix, modelization, school models, testimony

\section{AUTEUR}

\section{OLIVIA LEWI}

Paris-Sorbonne STIH (équipe Concepts et Langages) 